\title{
Decoding Algorithms for 3-D Space-Time-Frequency Codes
}

\author{
Pei $\mathrm{Xiao}^{\dagger}$, Jinsong $\mathrm{Wu}^{\dagger \dagger}$, Qingchun $\mathrm{Chen}^{\S}$ \\ ${ }^{\dagger}$ Centre for Communication Systems Research (CCSR) \\ University of Surrey, Guildford, Surrey, GU2 7XH, United Kingdom \\ E-mail: p.xiao@surrey.ac.uk \\ ${ }^{\dagger}$ Alcatel Shanghai Bell Company Ltd. \\ Pudong Jinqiao, Shanghai 201206, China \\ E-mail:wujs@ieee.org \\ $\S$ Key Lab of Information Coding and Transmission \\ Southwest Jiaotong University, Chengdu 610031, China \\ E-mail: qcchen@swjtu.edu.cn
}

\begin{abstract}
In this paper, we propose a joint complex diversity coding (CDC) and channel coding based space-time-frequency codes (STFCs) to increase diversity gains over space, time and frequency. Both non-iterative and iterative decoding of joint channel coding and 3-dimensional CDC transmission are investigated. The simulation results show that the minimum mean square error (MMSE) based iterative soft decoding achieves the performance of the soft sphere decoding (SD) with reduced complexity.
\end{abstract}

\section{INTRODUCTION}

Space-time-frequency coding (STFC) approaches have been recently investigated to exploit all the diversity available across space, time and frequency domains in [1]-[3]. The term complex diversity coding has been adopted to summarize diversity approaches using complex coding for more than one physical dimensional channel. Space-time-frequency codes are classified into seven different categories based on different integrations of complex diversity coding (CDC) and error control coding (ECC). Iterative decoding for ECC coded twodimensional full space diversity, full-rate (FDFR) system using the soft-to-hard sphere decoding (SHD-SD) scheme [4] is investigated in [5], [6]. However, to the best of our knowledge, the issue of iterative decoding for joint ECC and 3-D CDC based STFC has not been addressed in the existing literature. In this paper, a low complexity, high performance iterative MMSE inner decoding for the high rate 3-D CDC based STFC decoding and Log-MAP outer ECC decoding is proposed.

The notations used in this paper are summarized as follows: $(\cdot)^{\mathcal{T}}$ stands for matrix transpose, $(\cdot)^{\mathcal{H}}$ stands for matrix transpose conjugate, $E[\cdot]$ stands for expectation operation, $j$ is the square root of $-1, \mathbf{I}_{K}$ denotes identity matrix of size $K \times K, \mathbf{0}_{M \times N}$ denotes zero matrix of size $M \times N, \mathbf{A} \otimes \mathbf{B}$ denotes Kronecker (tensor) product of matrices $\mathbf{A}$ and $\mathbf{B}$, $[\mathbf{A}]_{a, b}$ denotes the $(a, b)$ entry of matrix $\mathbf{A}$, and $\operatorname{diag}(\cdot)$ transforms the argument from a vector to a diagonal matrix, and $\operatorname{vec}(\mathbf{X})$ denotes $\operatorname{vec}(\mathbf{X})=\left[\left[[\mathbf{X}]_{:, 1}\right]^{\mathcal{T}}, \ldots,\left[[\mathbf{X}]_{:, N}\right]^{\mathcal{T}}\right]^{\mathcal{T}}$, where matrix $\mathbf{X}$ is of size $M \times N$.

\section{PROPOSED SYSTEM MODEL}

A. space-time-frequency block (STFB) and space-timefrequency code (STFC)

We consider a MIMO-OFDM system with $N_{t}$ transmit antenna, $N_{r}$ receive antenna, and a block of $N_{c}$ OFDM subcarriers per antenna. Channel coefficients are assumed to be constant within one OFDM block, however, they may statistically vary from block to block, frequency selective order for each transmit-receive channel path is $L$. The proposed system could thus be used in dynamic environments. One CDC based STFC codeword contains $D$ space-time-frequency blocks (STFB), each of which is of size $N_{t} \times N_{F} \times T$, i.e. across $N_{t}$ transmit antennas $N_{F}$ subcarriers and $T$ OFDM blocks, where $N_{C}=$ $D N_{F}$. The data sequence is modulated using complex-valued symbols $\alpha_{q}+i \beta_{q}$, chosen from an arbitrary constellation (e.g., r-PSK or r-QAM). One STFB, denoted by $\mathbf{S}_{S T F B}$, can be written in matrix form as $\mathbf{S}_{S T F B}=\sum_{q=1}^{Q}\left(\alpha_{q} \mathbf{A}_{q}+j \beta_{q} \mathbf{B}_{q}\right)$, where $\mathbf{A}_{q} \in \mathbb{C}^{N_{T} \times N_{F} T}$ and $\mathbf{B}_{q} \in \mathbb{C}^{N_{T} \times N_{F} T}$ are dispersion matrices for the real and image parts of source signals. If $\mathbf{A}_{q}=\mathbf{B}_{q}$, vec $\left(\mathbf{S}_{S T F B}\right)=\mathbf{G}_{S T F B} \theta$, where $\mathbf{G}_{S T F B}=$ $\left[\operatorname{vec}\left(\mathbf{A}_{1}\right), \ldots, \operatorname{vec}\left(\mathbf{A}_{Q}\right)\right]$, and $\mathbf{s}=\left[s_{1}, \ldots, s_{Q}\right]^{\mathcal{T}}$.

The baseband frequency domain signal for the $i$-th STFB within the $k$-th STFC can be written as

$$
\mathbf{y}^{(i, k)}=\sqrt{\frac{\rho}{N_{t}}} \mathbf{H}_{S T F B}^{(i, k)} \mathbf{G}_{S T F B} \mathbf{S}^{(i, k)}+\mathbf{n}^{(i, k)},
$$

where $\mathbf{H}_{S T F B}^{(i, k)}$ is the corresponding frequency domain channel matrix of size $N_{r} N_{F} T \times N_{t} N_{F} T$. Both vectors $\mathbf{y}^{(i, k)}$ and $\mathbf{n}^{(i, k)}$ are of size $N_{r} N_{F} T$, and they are the frequency domain received signal and additive complex Gaussian noise vector, respectively. The source signal vector $\mathbf{s}^{(i, k)}$ is of size $N_{t} N_{F} T$. The channel matrix of the $i$-th STFB within the $k$-th STFC is $\mathbf{H}_{S T F B}^{(i, k)}$.

\section{B. System structure}

The ECC coded streams are first interleaved with random interleaver, and mapped into complex source symbols, which are subsequently encoded into CDC based STFCs. It is assumed 


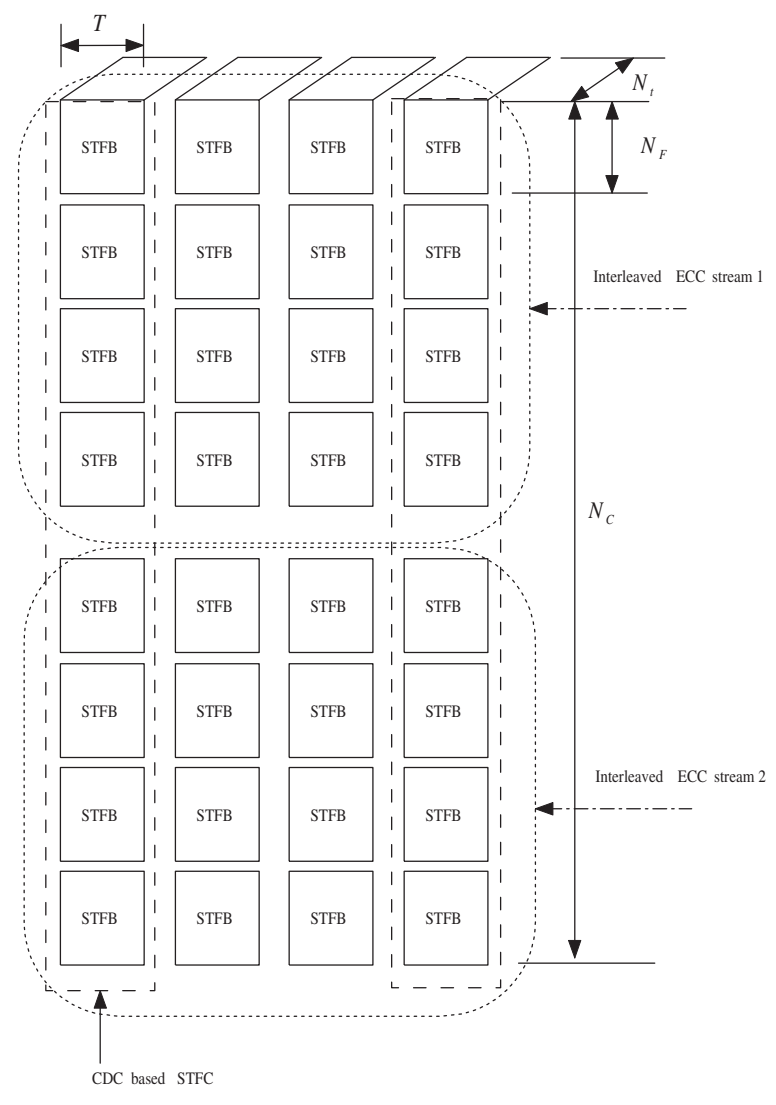

Fig. 1. The structure of the STFC code.

that one set of ECC streams for one joint CDC and channel coding block is across $K$ STFCs and $N_{a}$ STFBs within one STFC. Fig. 1 illustrates one example of the proposed system structure, which is used in our simulations, where $D=8, N_{r}=N_{t}=2, T=2, N_{F}=4, N_{a}=4, N_{C}=$ $D N_{F}=32$ in one STFC. Note that each 3-D CDC based STFC consists of 8 STFB. $\mathbf{G}_{S T F B}$ is unitary matrix of size $16 \times 16$, and $\mathbf{s}^{(i)}=\left[\left[\mathbf{s}_{1}^{(i)}\right]^{\mathcal{T}},\left[\mathbf{s}_{2}^{(i)}\right]^{\mathcal{T}}\right]^{\mathcal{T}}$, where $\mathbf{s}_{m}^{(i)}, m=1,2$, is the source symbol vector for the $m$-th thread of the $i$ th STFB. In this example, only one convolutional code is encoded in each block of joint CDC and ECC, as shown in each dashed rectangular area in Fig. 1. Iterative decoding may be applied within each block. Two joint CDC-ECC blocks are shown in the figure: the first one is across $\operatorname{STFB}_{i}^{(r)}$, $i=1, \ldots, 4, r=1, \ldots, 4$; the second one is across $\operatorname{STFB}_{i}^{(r)}$, $i=5, \ldots, 8, r=1, \ldots, 4$.

\section{DECODING OF JOINT CDC-ECC STFC}

Fig. 2 shows the diagram of the iterative joint CDC-ECC STFC decoding scheme. The STFC decoder takes channel observation vector $\mathbf{y}$ and a priori information $\lambda\left(c^{\prime} ; I\right)$ on the coded and interleaved bits $c^{\prime}$ and computes its extrinsic information $\lambda\left(c^{\prime} ; O\right)$, which is subsequently interleaved to $\lambda(c ; I)$. With a priori input $\lambda(c ; I)$, a soft-input, soft-output (SISO) ECC decoder computes log-likelihood ratio (LLR) $\lambda(c ; O)$ for the coded bits and $\lambda(b ; O)$ for the information bits. The latter is used at the final iteration to make hard decision on the transmitted information bits; whereas the former is interleaved and fed back to the STFC decoder as a priori information.
Several SISO algorithms can be applied to compute the ECC decoder output. For the purpose of this study, we consider the use of Log-MAP algorithm [8]. Although the iterative procedure as well as the Log-MAP decoder illustrated in Fig. 2 are quite standard, the STFC decoder has to be properly designed to achieve satisfactory performance and affordable complexity. The STFC decoding algorithms and the derivation of $\lambda\left(c^{\prime} ; O\right)$ will be explained next.

\section{A. Iterative MMSE decoding}

Optimal decoding scheme for the concatenated system under question should combine the trellises of both STF and EC codes, similar to the decoding of space-time trellis codes [9]. It certainly offers ML detection for the joint CDC-ECC STFC transmissions, but also results in a prohibitive computational complexity, which grows exponentially with the number of antennas and the number of data block length as well as the constraint length of the employed EC code. A practical suboptimal solution to reduce complexity and in the meantime, to approach the ML performance is Turbo processing, the principle of which is to perform successive soft decoding for each block through an iterative process. At each iteration, extrinsic information is extracted from one decoder and passed to another decoder as a priori information for the next iteration. In this section, we will show how turbo processing principle can be applied to the iterative STFC and ECC decoding.

Recall that the received signal vector is expressed as $\mathbf{y}=$ $\sqrt{\frac{\rho}{N_{t}}}$ HGs $+\mathbf{n}$. The transmitted symbol vector $\mathbf{s}$ can be estimated by a linear MMSE algorithm, i.e.,

$$
\mathbf{z}=\mathbf{W}^{H} \mathbf{y}=\mathbf{W}^{H}\left(\sqrt{\frac{\rho}{N_{t}}} \mathbf{H} \mathbf{G} \mathbf{s}+\mathbf{n}\right)=\mathbf{U} \mathbf{s}+\mathbf{v},
$$

where the matrix $\mathbf{W}$ is designed to minimize the mean square error $e=\mathrm{E}\left[\|\mathbf{z}-\mathbf{s}\|^{2}\right]$, leading to the solution $\mathbf{W}=\mathbf{R}^{-1} \mathbf{P}$, where

$$
\begin{aligned}
\mathbf{R} & =\mathrm{E}\left[\mathbf{y} \mathbf{y}^{H}\right]=\mathrm{E}\left[\frac{\rho}{N_{t}}(\mathbf{H G} \mathbf{\mathbf { n }})\left(\mathbf{s}^{H} \mathbf{G}^{H} \mathbf{H}^{H}+\mathbf{n}^{H}\right)\right] \\
& =\frac{\rho}{N_{t}} \mathbf{H} \mathbf{H}^{H}+N_{0} \mathbf{I} ; \\
\mathbf{P} & =\mathrm{E}\left[\mathbf{y} \mathbf{s}^{H}\right]=\mathrm{E}\left[\sqrt{\frac{\rho}{N_{t}}}(\mathbf{H G} \mathbf{s}+\mathbf{n}) \mathbf{s}^{H}\right]=\sqrt{\frac{\rho}{N_{t}}} \mathbf{H G} ; \\
\mathbf{U} & =\mathbf{W}^{H} \mathbf{P} .
\end{aligned}
$$

Equ. (3) is derived utilizing the fact that $\mathbf{G}$ is a unitary matrix. The noise term $\mathbf{v}$ is Gaussian since it is a linear transformation of a Gaussian random vector $\mathbf{n}\left(\mathbf{v}=\mathbf{W}^{H} \mathbf{n}\right)$. It has zero mean and covariance $\Gamma=\mathrm{E}\left[\mathbf{v v}^{H}\right]=N_{0} \mathbf{W}^{H} \mathbf{W}$. Because the filtered noise $\mathbf{v}$ is no longer white ( $\Gamma$ is not an identity matrix, the elements of $\mathbf{v}$ are correlated), optimum detection involves joint estimation of all the symbols in the vector $\mathbf{s}$, which requires $\mathrm{ML}$ or near-ML sphere decoding. However, we have observed from our experiments that the off-diagonal elements of $\Gamma$ are quite small compared to the diagonal elements. Therefore, we can approximate $\Gamma$ as a diagonal matrix. Consequently, each element of $\mathbf{s}$ can be estimated individually, and the receiver design is greatly simplified. The $k$ th element of $\mathbf{z}$, denoted by $z_{k}$, can be written as $z_{k}=u_{k} s_{k}+v_{k}$, where $u_{k}$ is the $k$ th diagonal element of $\mathbf{U}$, and $s_{k}, v_{k}$ are the $k$ th element of the vector $\mathbf{s}, \mathbf{v}$, respectively. The noise term $v_{k}$ is a Gaussian random variable with zero 


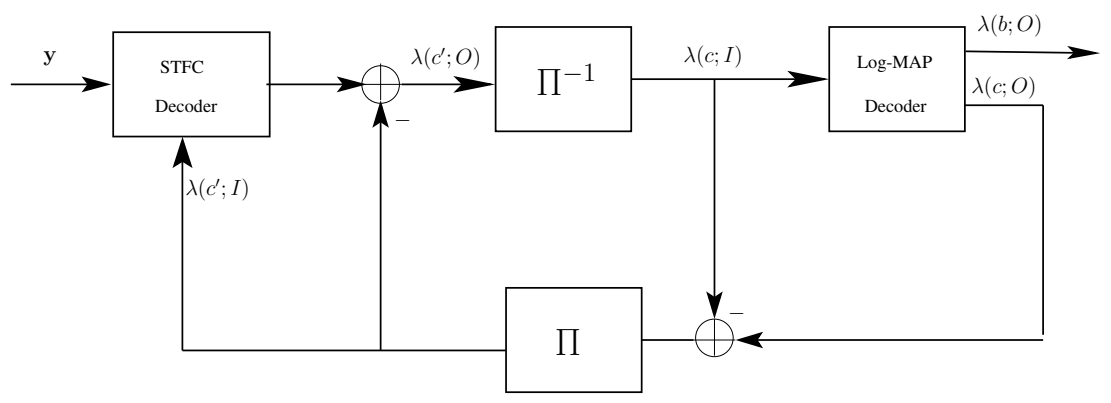

Fig. 2. Structure of the iterative STFC decoding.

mean and variance $N_{k}$, which is the $k$ th diagonal element of the matrix $\Gamma$. The probability density function (PDF) of the MMSE filter output $z_{k}$, conditioned on that the $m$ th PSK/QAM symbol is transmitted, can be expressed as

$$
f\left(z_{k} \mid s_{m}\right)=\frac{1}{\pi N_{k}} \exp \left(-\frac{\left|z_{k}-u_{k} s_{m}\right|^{2}}{N_{k}}\right) .
$$

In what follows, we derive a general expression for symbol to bit LLR mapping scheme based on the PDF function expressed by (5) for different modulation schemes. For a PSK/QAM system, we need to compute LLRs for $M$ coded bits for each symbol $s_{k}$, which is one of the $r=2^{M}$ possible symbols in the signal constellation. For example, $M=2$ for QPSK, and $M=4$ for 16-QAM. Denote the transmitted symbol $s_{k}=\left.\operatorname{map}\left\{c_{k}^{0 \prime}, c_{k}^{1 \prime}, \ldots, c_{k}^{(M-1) \prime}\right\}\right|_{c_{k}^{m \prime} \in\{0,1\}}$, the Lvalue of the bit $c_{k}^{m \prime}$ conditioned on the MMSE filter output $z_{k}$ can be calculate as

$$
\lambda\left(c_{k}^{m \prime} \mid z_{k}\right)=\ln \frac{\operatorname{Pr}\left(c_{k}^{m \prime}=1 \mid z_{k}\right)}{\operatorname{Pr}\left(c_{k}^{m \prime}=0 \mid z_{k}\right)} .
$$

To simplify (6), we define $\mathbf{I}_{+m}$ and $\mathbf{I}_{-m}$ for $m=0$ as

$$
\mathbf{I}_{-0}=\underbrace{\left(\begin{array}{ccccc}
0 & 0 & \cdots & 0 & 0 \\
0 & 0 & \cdots & 0 & 1 \\
\vdots & \vdots & \cdots & \vdots & \vdots \\
0 & 0 & \cdots & 1 & 1 \\
0 & 1 & \cdots & 0 & 1
\end{array}\right)}_{M \times 2^{M-1}} ; \quad \mathbf{I}_{+0}=\underbrace{\left(\begin{array}{ccccc}
1 & 1 & \cdots & 1 & 1 \\
0 & 0 & \cdots & 0 & 1 \\
\vdots & \vdots & \cdots & \vdots & \vdots \\
0 & 0 & \cdots & 1 & 1 \\
0 & 1 & \cdots & 0 & 1
\end{array}\right)}_{M \times 2^{M-1}}
$$

Note that for $m=0$, the first row of matrix $\mathbf{I}_{-0}$ has all elements equal to 0 , while the first row of matrix $\mathbf{I}_{+0}$ has all elements equal to 1 . The other matrices for $m=$ $1,2, \ldots, M-1$ can be found by exchanging the 1 st row with the corresponding $(m+1)$ th row. Using Bayes' theorem, we can write (6) as

$$
\lambda\left(c_{k}^{m \prime} \mid z_{k}\right)=\lambda\left(c_{k}^{m \prime}\right)+\ln \frac{\sum_{p=0}^{2^{M-1}} p\left(z_{k} \mid \operatorname{map}\left\{\mathbf{i}_{+m p}\right\}\right) e^{\mathbf{L} \mathbf{i}_{m p}}}{\sum_{p=0}^{2^{M-1}} p\left(z_{k} \mid \operatorname{map}\left\{\mathbf{i}_{-m p}\right\}\right) e^{\mathbf{L} \mathbf{i}_{m p}}},
$$

where $\mathbf{i}_{+m p}$ and $\mathbf{i}_{-m p}$ are $(p+1)$ th column vectors of matrices $\mathbf{I}_{+m}$ and $\mathbf{I}_{-m}$. Moreover, $\mathbf{i}_{m p}$ is the $(p+1)$ th column vector of matrix $\mathbf{I}_{+m}$ with its $m$ th entry set equal to zero, and $\mathbf{L}=$ $\left[\lambda\left(c_{k}^{0 \prime}\right) \lambda\left(c_{k}^{1 \prime}\right) \ldots \lambda\left(c_{k}^{(M-1) \prime}\right)\right]$ is a row vector of $a$ posteriori LLRs. The second term in (7) is the extrinsic information of bit $c_{k}^{m \prime}$. Denoting the extrinsic information of the $m$ th bit by $\lambda_{e}\left(c_{k}^{m \prime}\right)$, we have

$$
\lambda_{e}\left(c_{k}^{m \prime}\right)=\ln \frac{\sum_{p=0}^{2^{M-1}} p\left(z_{k} \mid \operatorname{map}\left\{\mathbf{i}_{+m p}\right\}\right) e^{\mathbf{L} \mathbf{i}_{m p}}}{\sum_{p=0}^{2^{M-1}} p\left(z_{k} \mid \operatorname{map}\left\{\mathbf{i}_{-m p}\right\}\right) e^{\mathbf{L} \mathbf{i}_{m p}}}
$$

Substituting (5) into (8) yields

$$
\begin{aligned}
\lambda_{e}\left(c_{k}^{m \prime}\right)= & \max ^{*}\left\{-\left|z_{k}-u_{k} \operatorname{map}\left\{\mathbf{i}_{+m 0}\right\} \mathbf{L} \mathbf{i}_{m 0}\right|^{2} / N_{k}, \ldots,\right. \\
& \left.-\left|z_{k}-u_{k} \operatorname{map}\left\{\mathbf{i}_{+m(P-1)}\right\} \mathbf{L} \mathbf{i}_{m(P-1)}\right|^{2} / N_{k}\right\} \\
- & \max ^{*}\left\{-\left|z_{k}-u_{k} \operatorname{map}\left\{\mathbf{i}_{-m 0}\right\} \mathbf{L i}_{m 0}\right|^{2} / N_{k}, \ldots,\right. \\
& \left.-\left|z_{k}-u_{k} \operatorname{map}\left\{\mathbf{i}_{-m(P-1)}\right\} \mathbf{L} \mathbf{i}_{m(P-1)}\right|^{2} / N_{k}\right\},
\end{aligned}
$$

where $\max ^{*}[]$ is defined as $\max ^{*}[x, y]=\ln \left(e^{x}+e^{y}\right)=$ $\max [x, y]+\ln \left(1+e^{-|x-y|}\right)$.

In Section IV, this MMSE solution is compared with the soft sphere decoding (SD) scheme presented in [4]. SD is shown to have an exponential complexity (in the worst case as well as in the average case) of $O\left(r^{\gamma N_{b}}\right)$ with $\gamma \in(0,1]$ ( $\gamma$ decreases as SNR increases) [10], [11], where $r$ is the constellation size. In order to ensure a certain probability of finding a point within the sphere, the radius of the sphere must grow with the problem size, and consequently, the number of operations required by sphere decoder always grows as an exponential function of the problem size [10]. The situation will be exacerbated if an iterative process is to be employed. One can see from (4) that the complexity of the proposed MMSE scheme mainly comes from the matrix inversion, and compared to sphere decoding, it reduces the complexity from exponential $O\left(r^{\gamma N_{b}}\right)$ to polynomial $O\left(N_{b}^{3}\right)$.

\section{Numerical RESUlts}

In the simulations, we mainly employ a rate $R_{c}=1 / 2$ convolutional code. Two bit-to-symbol mapping schemes, namely, Gray and anti-Gray are considered in this work, for QPSK and 16-QAM systems, respectively. Other simulation settings are summarized as follows 1) MIMO frequency selective channel has channel order $L=1$ ( 2 paths), and each path experiences independent Rayleigh fading. Channel power delay profile is assumed to be uniform. 2) $N_{t}=N_{r}=2, N_{F}=4, T=2$, and $N_{C}=32$.

Performance of different decoding algorithms for the joint CDC-ECC system with QPSK modulation are demonstrated in Fig. 3. Comparing the two non-iterative schemes, soft sphere decoding (SD) shows $1 \mathrm{~dB}$ gain at $\mathrm{BER}=10^{-4}$ compared to the MMSE scheme with gray mapping. However, we observed that the performance of the MMSE decoding can be much improved by using anti-gray mapping and iterative decoding, which is slightly better than or comparable to the non-iterative soft SD decoding over a wide range of SNRs.

Fig. 4 shows the convergence behavior of the iterative MMSE decoding for QPSK modulated joint CDC-ECC STFC system with anti-gray mapping. Apparently, it is significant 


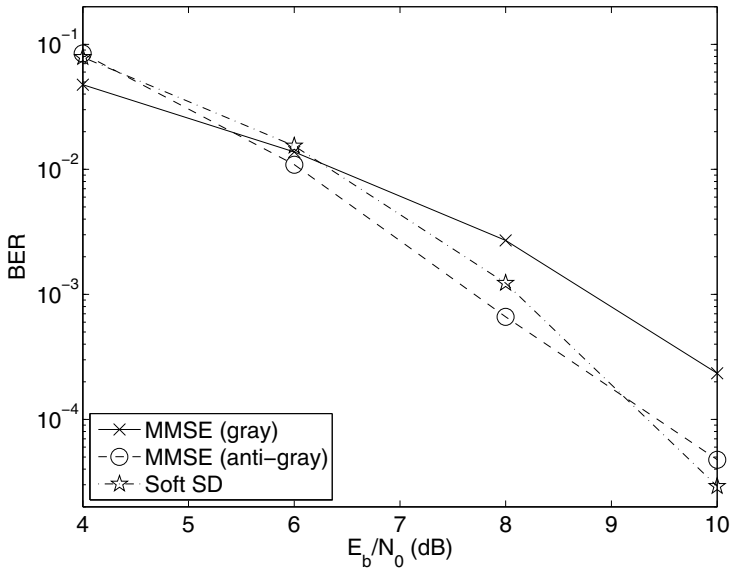

Fig. 3. Performance comparison of MMSE and sphere decoding in ECC STFC system with QPSK modulation. MMSE with gray mapping and soft SD decoding schemes are non-iterative. The curve for MMSE with anti-gray mapping is plotted at the 4th iteration.

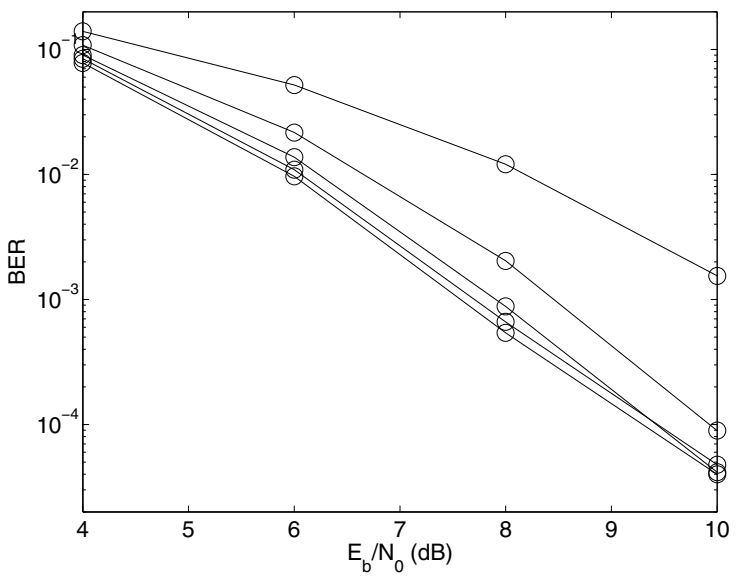

Fig. 4. Performance of iterative MMSE decoding for QPSK system.

performance improvement by applying iterative process if we compare the topmost curve representing the first-iteration CDC based STFC MMSE decoding and Log-MAP ECC decoding with the bottom curve representing the performance of iterative decoding upon convergence. It takes 3 iterations for the algorithm to reach steady state, and further iterations do not yield noticeable performance improvement. The most significant gain is obtained at the second iteration. Note that no gain can be obtained by performing iterative process for the systems with gray mapping, in which the bits are mapped to I and Q channels independently [12].

Fig. 5 shows the performance of the MMSE scheme for the 16-QAM system. The system with gray-mapping is noniterative. The iterative MMSE decoding with anti-gray mapping outperforms the one with gray mapping at the 3rd iteration when $E_{b} / N_{0}>20 \mathrm{~dB}$, which suggests that if the 16-QAM system operates at low SNR, gray mapping can be applied; otherwise, anti-gray mapping and iterative decoding can be applied to improve the system performance.

\section{CONCLUSIONS}

We have investigated a joint complex diversity coding and channel coding to increase diversity across space, time and

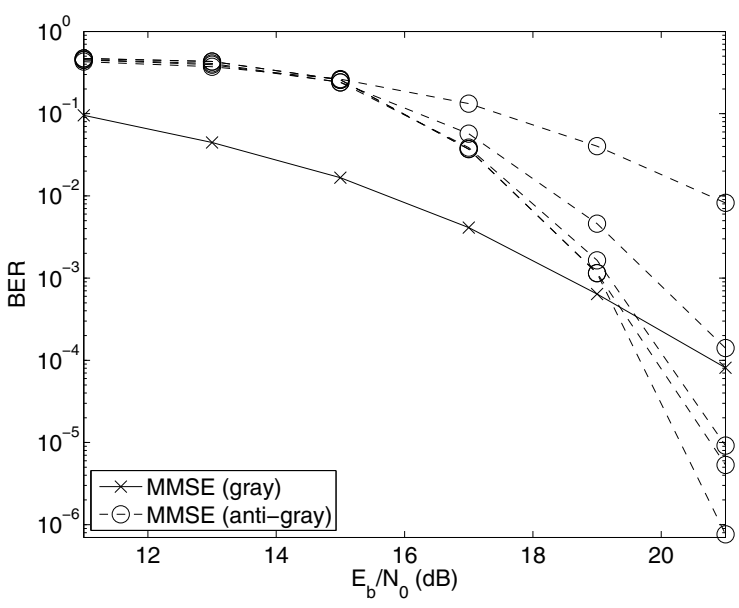

Fig. 5. Performance of iterative MMSE decoding for 16-QAM system.

frequency in this paper. A low complexity MMSE iterative decoding of the joint ECC and CDC has been proposed to exploit the diversity potential, and has been shown to achieve the performance of the non-iterative soft sphere decoding with exponential complexity.

\section{ACKNOWLEDGEMENT}

Qingchun Chen's work is partly supported by the NSFC under grant no. 60872013 and the the Fundamental Research Funds for the Central Universities under grant no. SWJTU11CX043.

\section{REFERENCES}

[1] Z. Liu, G. Giannakis. "Space-time-frequency coded OFDM over frequency-selective fading channels". IEEE Trans.on Sig. Proc., vol. 50, no. 10, pp. 2465-2476, Oct. 2002.

[2] W. Zhang, X. Xia, P. Ching. "High-rate full-diversity space-timefrequency codes for broadband MIMO block-fading channels". IEEE Trans. on Commun., vol. 55, no. 1, pp. 25-34, Jan. 2007.

[3] J. Wu and S. Blostein "Improved high-rate space-time-frequency block codes". Proc. IEEE Globecom, pp. 1-5, Nov. 2006.

[4] R. Wang, G. Giannakis. "Approaching MIMO channel capacity with reduced-complexity soft sphere decoding". Proc. IEEE WCNC, vol. 3, pp. 1620-1625, March 2004.

[5] Z. Wang, S. Zhou, G. Giannakis. "Joint coding-precoding with lowcomplexity turbo-decoding”. IEEE Trans. on Wireless Commun., vol. 3, no. 3, pp. 832-842, May 2004.

[6] R. Wang, X. Ma, G. Giannakis. "Improving the performance of coded FDFR multi-antenna systems with turbo-decoding". Wireless Communications and Mobile Computing, vol. 4, pp. 711-725, 2004.

[7] S. Siwamogsatham, M. Fitz, J. Grimm. "New view of performance analysis of transmit diversity schemes in correlated Rayleigh fading". IEEE Trans. Inform.Theory, vol. 48, no. 4, pp. 950-956, Apr. 2002.

[8] P. Robertson, P. Hoeher, E. Villebrum. "Optimal and sub-optimal maximum a posteriori algorithms suitable for Turbo decoding". European Transactions on Telecommunications, vol. 8, no. 2, pp. 119-125, MarchApril, 1997.

[9] V. Tarokh, N. Seshadri, A. Calderbank. "Space-time codes for high data rates wireless communications: performance criterion and code construction". IEEE Trans. on Inform. Theory, vol. 44, pp. 744-765, March 1998.

[10] J. Jalden, B. Ottersten. "On the complexity of sphere decoding in digital communicationsg”. IEEE Trans. on Sig. Proc., vol. 53, no. 4, pp. 1474 1484, April 2005.

[11] J. Jalden, L. barbero, B. Ottersten, J. Thompson. "Full diversity detection in MIMO systems with a fixed-complexity sphere decoder". Proc. ICASSP '07, vol. 3, pp. 49-52, April 2007.

[12] S. Brink, J. Speidel, R. Yan. "Iterative demapping for QPSK modulation”. Electronic Letters, vol. 34, no. 15, pp. 1459-1460, July 1998. 